\title{
Power grid disturbances and polar cap index during geomagnetic storms
}

\author{
Peter Stauning \\ Danish Meteorological Institute, Lyngbyvej 100, DK-2100 Copenhagen, Denmark \\ Corresponding author: e-mail: pst@dmi.dk
}

Received 13 March 2013 / Accepted 22 May 2013

\begin{abstract}
The strong geomagnetic storm in the evening of 30 October 2003 caused high-voltage power grid disturbances in Sweden that expanded to produce hour-long power line outage in Malmö located in the southern part of the country. This was not a unique situation. The geomagnetic storm on 13 March 1989 caused extensive disruptions of high-voltage power circuits especially in the Province of Quebec, Canada, but also to a lesser degree in Scandinavia. Similar events have occurred earlier, among others, during the great storms of 13-14 July 1982 and 8-9 February 1986. These high-voltage power grid disturbances were related to impulsive magnetic variations accompanying extraordinarily intense substorm events. The events were preceded by lengthy intervals of unusually high values of the Polar Cap (PC) index caused by enhanced transpolar ionospheric convection. The transpolar convection transports magnetic flux from the dayside to nightside which causes equatorward displacements of the region of auroral activity enabling the substorms to hit vital power grids. During the 30 October 2003 event the intense solar proton radiation disabled the ACE satellite observations widely used to provide forecast of magnetic storm events. Hence in this case the alarmingly high PC index could provide useful warning of the storm as a back-up of the missing ACE-based forecast. In further cases, monitoring the PC index level could provide supplementary storm warnings to the benefit of power grid operators.
\end{abstract}

Key words. geomagnetically induced currents (GIC) - technological systems - magnetic fields - substorms - polar Ionosphere

\section{Introduction}

\subsection{Power grid disturbances}

On 29 October 2003 at 06:10 the Earth's magnetosphere was hit by the intense solar wind cloud from a major outburst, a Coronal Mass Ejection (CME), from the Sun, which in the following minutes caused world-wide Sudden Commencement (SC) impulses in the geomagnetic field. The SC was followed by an intense magnetic storm with a ring current index peaking at $D$ st $=-383$ on 30 October at 22 UT. The magnetic activity in the auroral zones as recorded through the Auroral Electrojet indices, notably $\mathrm{AL}$ and $\mathrm{AE}$, was very high through the stormy days. Preceding the impact of the solar wind cloud the Sun emitted an intense solar proton radiation which disabled several instruments on the interplanetary satellites, notably the ACE satellite, which were in use to provide forecast of the arrival of the intense solar wind. Hence many of the routine Space Weather forecast systems were not functioning properly. Such intense high-energy solar proton fluxes are commonly accompanying the largest solar outbursts.

In Scandinavia the substorm activity was very intense in the evenings of 29 and 30 October. At a major substorm outburst a little past 20:00 UT on 30 October, the power grid in Sweden was disturbed such that Malmö was hit by an hour-long electric power line outage. This event shall be the focus of the present work. However, it was not the only case of power grid disturbances in Sweden. On 13 March 1989, the day of the large power outage in Quebec, two series of disrupts of the $130 \mathrm{kV}$ high-voltage power lines occurred in Sweden. The first series occurred at 0744 UT in the morning $(\approx 0900$ local time) while the other series occurred at 2143 UT $(\approx 2300$ LT $)$ in the evening. In both cases the management of the power grid by the Swedish power company, "Vattenfallet", prevented further adverse consequences of the high-voltage power line cuts. The effects on consumers in Sweden were small and no effects were recorded in the coupled power grids in the neighboring countries, Finland, Norway, and Denmark during these storms (e.g., Stauning 2001; Stauning \& Watermann 2002; Wik et al. 2008, 2009).

\subsection{Auroral ionospheric currents and substorm activity}

Such disturbances on power grid systems are caused by voltages and currents related to electric fields induced at ground level by variable currents in the upper ionized atmosphere, the ionosphere, at altitudes of $100-150 \mathrm{~km}$. These currents are particularly intense in the auroral oval whose midnight sectors form the auroral zone. The ionospheric currents at the morning and evening sectors of the auroral oval are related to field-aligned currents generated in the boundary regions between the Earth's magnetosphere and the solar wind plasma streaming past the Earth at velocities of several hundred $\mathrm{km} / \mathrm{s}$. The most intense ionospheric currents are generally found in the night sector of the auroral oval and are driven by bursty field-aligned currents from substorm processes in the unstable magnetospheric tail region. The solar wind flow, and its interaction with the Earth's environment, can be strongly enhanced following CMEs. The interaction is particularly strong when the Interplanetary Magnetic Field (IMF) has a strong southward component. The total voltage induced across the magnetosphere may amount to several hundred $\mathrm{kV}$. The total intensity 


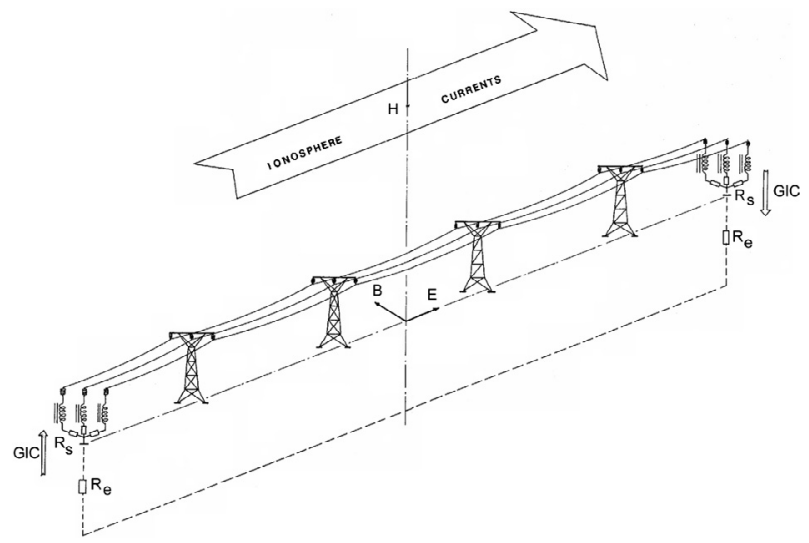

Figure 1. Sketch of power transmission line and the associated transformer and grounding circuits.

of the field-aligned currents flowing along the geomagnetic field connecting between the outer magnetosphere or the tail region and the ionosphere may amount to several million Ampères. The field-aligned currents close in the ionosphere through conduction (Pedersen) currents while strong transverse (Hall) currents are generated by the associated electric fields.

\subsection{Ionospheric currents and GIC events}

Since the atmosphere is a good insulator these field-aligned and ionospheric currents will not directly reach ground level. However, variable currents in space generate magnetic variations and thus induce secondary electric fields at ground level. These, in turn, drive Geomagnetically Induced Currents (GIC) in conducting structures like high-voltage power transmission lines connected to different points of the Earth. The induced voltages along a power transmission line will drive currents along the line and through the windings of connected power transformers to the grounded null. The situation is sketched in Figure 1 from Stauning (2001).

The relations between ionospheric currents and the geoelectric fields at ground level are quite complex and shall not be further elaborated here (see, e.g., Boteler et al. 1998; Pirjola 1998; Pirjola \& Viljanen 1998; Viljanen et al. 1999). Ionospheric currents are three-dimensional quantities having complex temporal and spatial variations. Ground conductivity composition is very complicated. Power grids, in reality, are constructed as twodimensional networks with grounding connections at many grid points. However, it is possible (but complicated) to model GIC effects in real power systems with adequate accuracy (e.g., Viljanen \& Pirjola 1989; Pulkkinen et al. 2005; Wik et al. 2008, 2009). For the present purpose it suffices to state that the geoelectric field strengths and thus the intensities of GIC depend on the strength and variability of the ionospheric currents - in the simplest approximation expressed through the time derivative $\mathrm{d} \boldsymbol{B} / \mathrm{d} t$ of the variable magnetic field generated at ground level by the ionospheric currents (e.g., Viljanen et al. 2001).

Compared to power line frequencies $(50 / 60 \mathrm{~Hz})$ these currents, which typically vary at periods ranging from seconds to minutes, act as DC bias currents and may cause transformer unbalance. A transformer heavily loaded by its normal AC currents could be driven into saturation in one $\mathrm{AC}$ half-cycle phase by additional DC bias with subsequent waveform distortion, power loss, and heating in its core as the adverse results. If not guarded by protective circuits the transformer can be permanently damaged and eventually face burn-out during events with strong GICs. Further types of protective circuits for lines or for phase-correcting capacitor banks react on indications of over-voltage, unbalanced null currents, or other consequences of GIC events and may also cut the power circuits.

Disrupts of power transmission circuits are the most evident, but not the only consequence of GIC events. Unbalanced transformers with partially saturated cores increase the reactive component and the content of harmonics of the power supplied from power plants. Hence the power distribution efficiency is reduced whereby the power available to the consumers from a given generator setup may decrease. In order to avoid situations where the available power is inadequate to meet the consumption it could be required to start additional generators or even open further power plants during occurring or anticipated GIC events. Such conditions, in any event, increase the complexity and costs of power grid management. In worst cases the power grids may become unstable and break down causing large-scale power outages (e.g., Boteler et al. 1998; Kappenman 2010).

These conditions apply most often to regions poleward of approximately $50^{\circ}$ geomagnetic latitude such as the Scandinavian countries, UK, Canada, and Northern USA. However, there is a growing concern that really intense solar CME events, like the Carrington event in September 1859, might displace the region of strong GIC events further equatorward to hit power grids in countries like Germany and France and most of USA or Russia. The electric power management companies in these regions far away from the auroral zone may not be well enough prepared to deal with such, for them, unusual events (e.g., Kappenman 2010; Thomson et al. 2011).

\section{PC index definition and application}

\subsection{Basics}

The Polar Cap (PC) index was originally introduced by Troshichev \& Andrezen (1985) as a measure of the interplanetary electric field derived from available polar ground-based magnetic observations. The PC index concept was further developed by Troshichev et al. (1988) and Vennerstrøm (1991).

The basic definition of the PC index is derived from an assumed linear relation between the "geo-effective" (or "merging") electric field, $E_{\mathrm{M}}$, in the solar wind encountering the Earth and $\Delta F_{\text {proj, }}$ the polar cap magnetic magnetic variation (at ground) projected to the so-called optimum direction.

$$
\Delta F_{\text {proj }}=\alpha E_{\mathrm{M}}+\beta
$$

The optimum direction is the direction perpendicular to the average DP2 transpolar equivalent current direction and makes an angle $\varphi$ to the dawn-dusk direction.

The "geo-effective" (or "merging") electric field (Kan \& Lee 1979) is:

$$
E_{\mathrm{M}}=M E F=V_{\mathrm{SW}} B_{\mathrm{T}} \sin ^{2}\left(\frac{\theta}{2}\right),
$$

where $V_{\mathrm{SW}}$ is the solar wind velocity, $B_{\mathrm{T}}$ is the transverse component of the IMF $\left(B_{\mathrm{T}}=\sqrt{ }\left(B_{Y}^{2}+B_{Z}^{2}\right)\right.$, while $\theta$ is the polar angle between the $Z$-axis of a Geocentric Solar-Magnetospheric coordinate system and the transverse IMF component. 
The Eq. (1) is inverted to give a definition of the PC index by equivalence to the electric field measured in $\mathrm{mV} / \mathrm{m}$ :

$$
\mathrm{PC}=\left(\Delta F_{\text {proj }}-\beta\right) / \alpha \quad\left(\sim E_{\mathrm{M}} \text { in } \mathrm{mV} / \mathrm{m}\right)
$$

The scaling parameters used to derive the PC index from geomagnetic variations comprise the optimum direction angle, $\varphi$, and the regression coefficients, $\alpha$ and $\beta$. They are found from Eq. (1) through statistical analyses based on ensembles of concurrent values of the merging electric field, $E_{\mathrm{M}}$, and the $\mathrm{PC}$ horizontal magnetic variation vector, $\Delta \mathbf{F}$, counted from the quiet level, $\mathbf{F}_{\mathrm{QL}}$.

\subsection{Different $P C$ indices}

PCN (North) indices are based on measurements made at Thule in Greenland, while PCS (South) indices are based on measurements of magnetic variations at Vostok in Antarctica. At the Arctic and Antarctic Research Institute (AARI) the first set of PCS index values was derived in 1988 (Troshichev et al. 1988). In 1997 another set of coefficients was derived and then used for some years for calculations of AARI PCS indices. The first published set of coefficients for PCN based on Thule magnetic data was derived by Vennerstrøm (1991) and index values were currently made available. PCN indices derived using this set of coefficients are now supplied from DTU Space, among other, through the OMNIweb data service. Errors in the program used to calculate index values were corrected in 1999 and again in 2001. Hence PCN values published prior to 2001 are in error for this reason. In addition there are questionable features (Stauning 2011) in the original program used to calculate the coefficients still in use at DTU Space.

In 2005 a new "unified" procedure was introduced and sets of coefficients for PCS and PCN were derived at AARI (Troshichev et al. 2006). In 2006 Stauning at DMI adopted with small modifications the unified procedure proposed by Troshichev et al. (2006) for calculations of PCN and PCS indices. Index values calculated by this modified procedure (Stauning et al. 2008) are used in the present work.

In general the PCN and PCS index values calculated now by AARI and by DMI are similar in magnitude. The PCN index values calculated by DTU Space are considerably smaller in amplitude during disturbed conditions than the AARI or DMI index values. For the use of PC indices in forecast tasks the differences in derivation procedures are not important. The availability of PC index values relies on the availability of the basic magnetic data from Thule and Vostok. Both stations have established on-line data transfer to the responsible institutes. Hence $\mathrm{PC}$ index values can be available in real time.

The development of a final PC index was recommended by the International Association for Geomagnetism and Aeronomy (IAGA) in 1999 and the index was later adopted by IAGA on the condition that the derivation of PC-S (South) and PC-N (North) indices was unified, and a joint procedure for the $\mathrm{PC}$ index calculations was properly defined and published. However, series of PC indices based on the different procedures are still made available and used in publications. IAGA is expected to address the question of approval of a final procedure for PCN and PCS index derivation at its General Assembly to be held in August 2013.

\subsection{Coupling between PC index and substorm activity}

The PC indices, as outlined above, are derived from magnetic variations recorded within the polar caps. These variations are caused by sunward currents associated with the antisunward convection of ionospheric and magnetospheric plasma and embedded geomagnetic field over the polar caps. The transpolar convection, in turn, is driven by the dawn-dusk electric field resulting from the coupling between the auroral regions and the solar wind electric field. The PC index is scaled to be equivalent to the solar wind geo-effective (or "merging") electric field. Thus the PC index could be taken to indicate the magnitude of the solar wind electric field subtended across the Earth's magnetosphere.

This electric field is also acting across the magnetospheric tail region causing a sunward (earthward) motion of the tail plasma towards the night auroral region. This convection is potentially unstable and may occur in violent jumps causing magnetospheric and ionospheric substorm events. The transpolar convection of plasma and embedded magnetic fields detectable through the PC index add to increase the stresses in the tail region whereby substorm intensities are enhanced. A continuing high PC index level is indicative of a deformation of the magnetospheric structure whereby the auroral regions are displaced equatorward. This displacement seems to further enhance the intensity and variability of substorms. Thus enhancements of the PC index in three ways indicate the buildup of conditions that may result in violent substorm activity to relieve the stresses in the magnetospheric tail region and cause intense magnetic disturbances at ground level (e.g., Troshichev et al. 1979; Janzhura et al. 2007; Stauning et al. 2008; Stauning 2012). In coarse terms, a PC index level below 2 indicates quiet conditions. At PC index values between 2 and 5 substorm activity may buildup within an hour (e.g., Janzhura et al. 2007). When the PC index level exceeds 5 then substorm activity is imminent with strength and equatorward displacement related to the magnitude and duration of the enhanced index level. The equatorial displacement of auroral activity is a prerequisite for substorms to harm vital power grids in the European sector, in USA (except Alaska), and in the southern part of Canada.

\section{Selected geomagnetic storms}

\subsection{Summary of selected storms}

Observed geomagnetic variations and related power grid disturbances in Sweden have been examined for selected major geomagnetic storm events. These are:

- 13-14 July 1982. Post solar max. event,

- 8-9 February 1986. Solar minimum event,

- 13-14 March 1989. Solar maximum event,

- 30-31 October 2003. Post solar max. event.

For an analysis of these GIC events we have combined essential magnetic data in the diagrams in Figure $2 \mathrm{a}-\mathrm{d}$. The upper panels in the diagrams display the $\mathrm{PCN}$ indices during the storm events. The middle panels display the Upper (AU) and Lower (AL) Auroral Electrojet indices during the events. It should be noted that the Auroral Electrojet, AE, index is the difference between the AU and AL index values. The lower panels in the diagrams display the horizontal magnetic component at Lovö in Mid-Sweden located at an invariant latitude of $56.4^{\circ}$. It could be noted that this position is also midway between the large hydro-electric power plants in Northern Sweden and the body of consumers in Southern Sweden. In these diagrams 
(a)
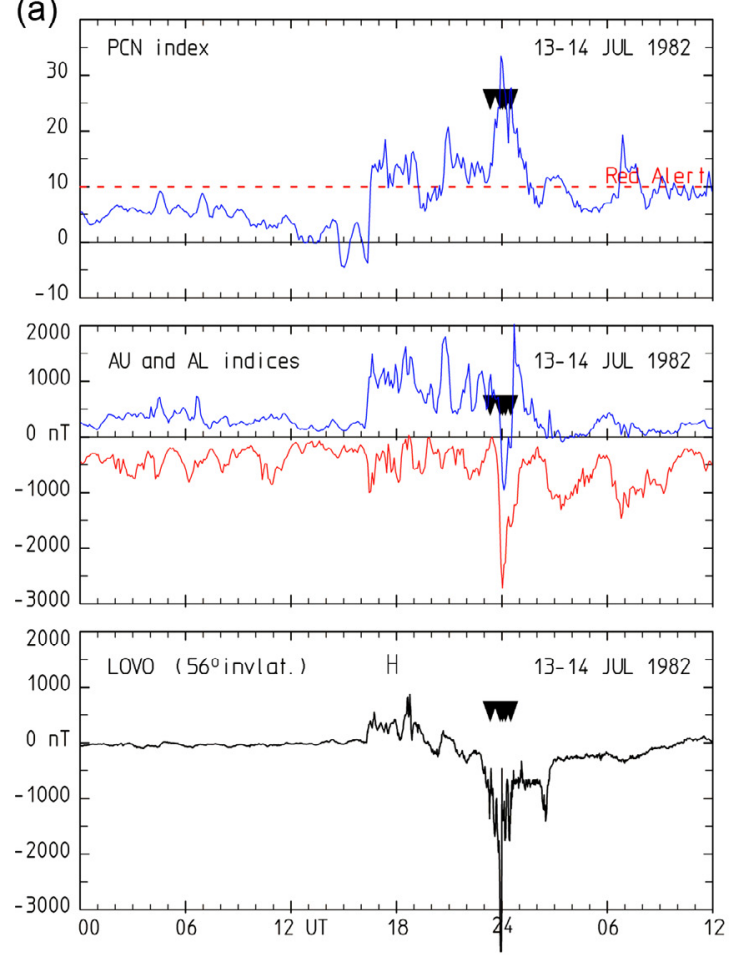

(c)
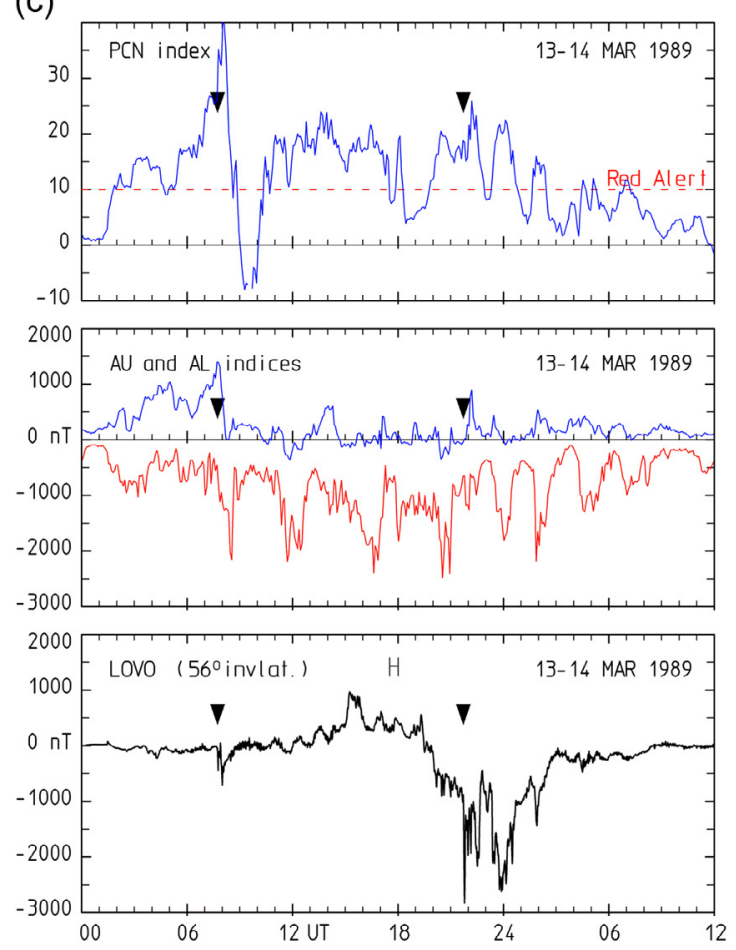

(b)
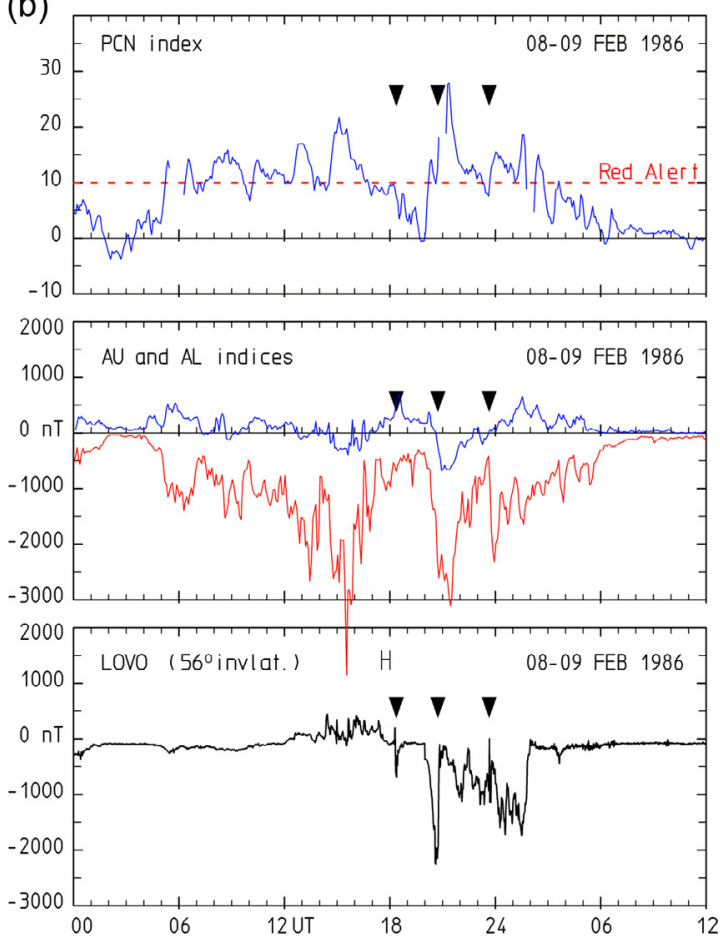

(d)
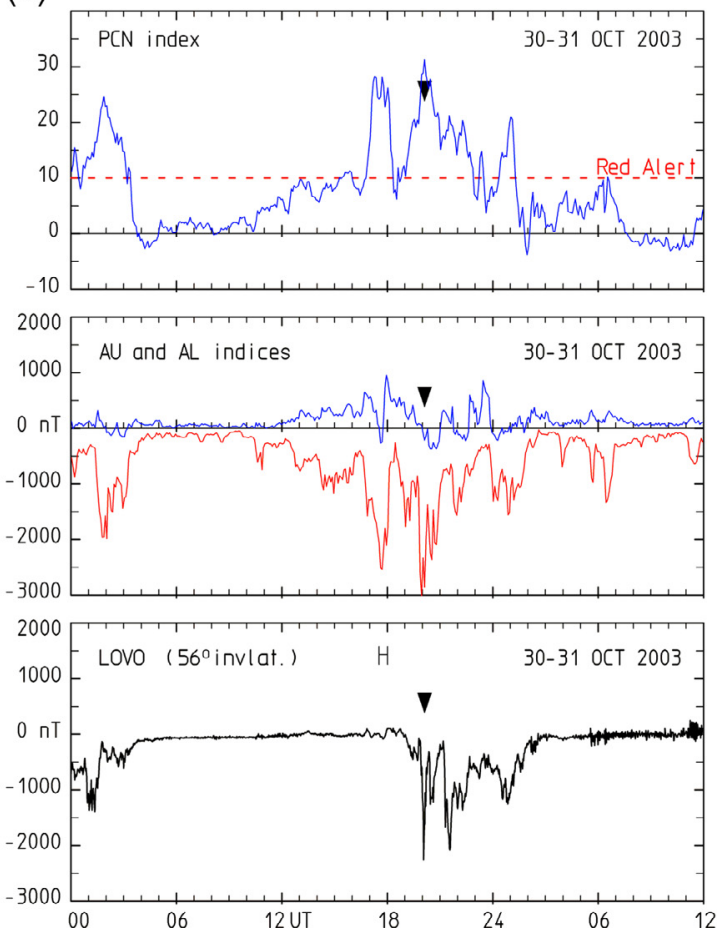

Figure 2. Polar Cap PCN indices, Auroral Electrojet AU and AL indices, and Lovö horizontal magnetic recordings during storms (a) 13-14 Jul. 1982, (b) 08-09 Feb. 1986, (c) 13-14 Mar. 1989, and (d) 30-31 Oct. 2003.

the occurrences of high-voltage power grid disturbances are indicated by the arrowhead signatures.

Several features of the displays in Figure 2 are noteworthy. Firstly, it is clear that all events without exception are accompanied by sudden and violent changes in the Lovö geomagnetic recordings. This is a reliable indication that the power grid disturbances are GIC-related. Furthermore, most of the events occur at or close to local midnight ( 23:00 UT). They are most often related to violent changes in the Auroral Electrojet, AL, index indicative of substorm occurrences. However, there are exceptions, for instance, at the first event at during 8-9 February 1986 and the second event of 13-14 March 1989. In these cases the AL indices are not very disturbed. The Auroral Electrojet indices are built from the magnetic recordings 
P. Stauning: Power grid disturbances and polar cap index during geomagnetic storms
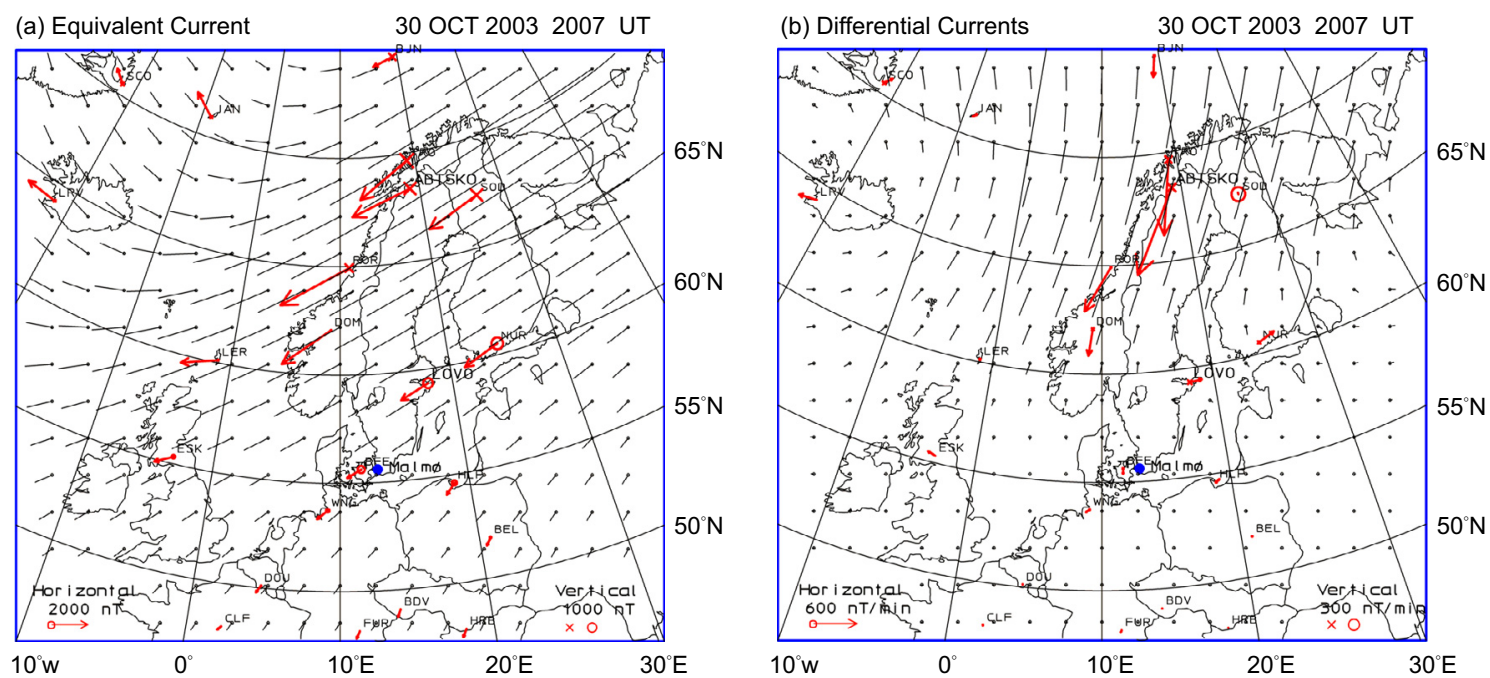

Figure 3. Substorm event at 20:07 UT on 30 October 2003. (a) Equivalent current patterns at 20:07. (b) Differential current patterns change from 20:06 to 20:07 UT. Note the different scales in the two diagrams.

from 10 to 12 observatories distributed in the northern (mostly) and southern auroral regions. They may not catch every single, possibly quite localized, disturbance.

An important observation is the occurrence of high levels of the polar cap, PCN, index values preceding and during all events. The high level of the PCN index indicates a situation where the stresses in the tail region are built up by the transpolar convection of plasma and embedded magnetic fields from the dayside of the magnetosphere to the nightside. These stresses were released in the violent substorms and associated strong ionospheric currents that caused the GIC disturbances. Furthermore, the extended duration of the high PC index level is indicative of the equatorward displacement of substorm activity enabling the associated magnetic disturbances to hit the power grids in Sweden. Hence, monitoring the PC index level may provide a forecast of possible GIC-related power grid disturbances. In Figure 2, a level of PCN $=10$ units (red dotted line) has been inserted to indicate alert conditions.

From a closer examination of the power grid disrupts marked in Figure 2, three different types emerged based on information supplied by the Swedish power company, "Vattenfallet":

1. tripping of line protective circuits,

2. tripping of capacitor protective circuits,

3. tripping of transformer protective circuits.

The first two failure modes may be considered "false trippings" since the GIC effects would probably harm neither the lines nor the phase-correcting capacitor banks. The third group, however, reflects the necessary protection against system damage, which could be caused by excessive heating of the transformer by possible transformer half-cycle core saturation when loaded by GIC currents.

\subsection{The magnetic storm on 29-30 October 2003}

In cases (a), (b), and (c) of the events displayed in Figure 2 the power company managed to avoid disrupts of the consumer electric power supply in spite of the high-voltage power grid disturbances. However, during case (d) the electric power to
Malmö, the largest city in Southern Sweden, experienced an hour-long electric power outage starting at 20:07 UT (21:07 local time) on 30 October 2003. Hence this event shall be given a closer examination.

There is no doubt that the power grid disturbances are related to sudden changes in the geomagnetic field caused by strong variations in the substorm-associated ionospheric currents that may amount to several millions of Ampères. But there may also be some dependence on the location of such currents and their orientation with respect to the power grid lines. The magnetic data from observatories in Scandinavia and other places in Northern Europe enable mapping of the auroral ionospheric current system and its changes around the time of the power grid failure on 30 October 2003.

For illustration, the ionospheric equivalent current system as derived from geomagnetic measurements at 20:07 on 30 October 2003 is displayed in the left diagram of Figure 3. The red arrows display the observed horizontal magnetic disturbances rotated $90^{\circ} \mathrm{CW}$ to indicate the directions and intensities of the local overhead currents. The cross (downward) or circle (upward) signatures display the disturbance in the vertical magnetic component which may contribute to indicate the location of the auroral electrojet current between upward and downward vertical disturbances. Signature scales (in nT) are noted at the bottom of the diagram. The streamline-like signature, line bars starting from regularly spaced "dots", have been derived by interpolation using also the available observations outside the displayed European segment.

It is seen from Figure $3 a$ that the currents are particularly strong within a northeast-to-southwest oriented strip over Northern Finland and Sweden and Mid-Norway. The right diagram of Figure 3 displays the time derivatives $\mathrm{d} \boldsymbol{B} / \mathrm{d} t$ rotated $90^{\circ}$ $\mathrm{CW}$ to indicate the change in equivalent currents from 20:06 to 20:07 UT. Note the signature scales at the bottom of Figure $3 \mathrm{~b}$ (in $\mathrm{nT} / \mathrm{min}$ ). It is seen that the major change is a strong intensification of the southward current component over Northern Sweden. This orientation gives maximum inductive effect on the power grid lines running North-to-South in Sweden.

Figure 4 provides a closer view of the relations between the PC index and geomagnetic variability. At the first enhancement of PCN in Figure 4 during around 17-18 UT, Scandinavia is 


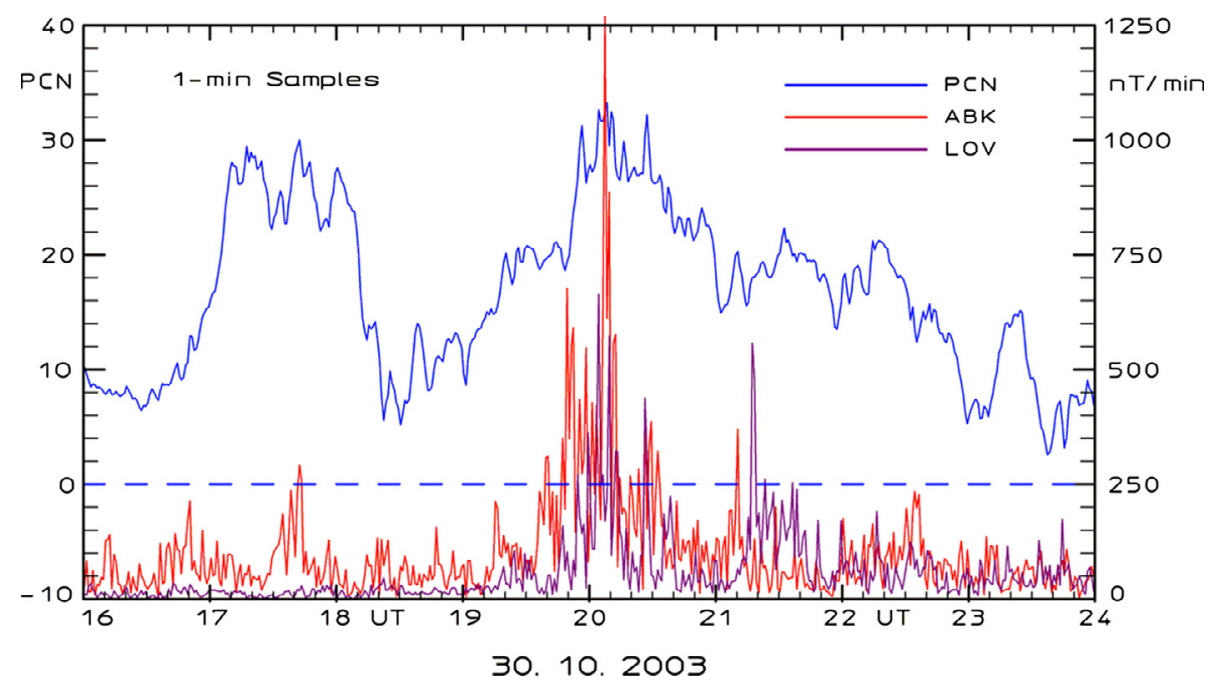

Figure 4. Polar Cap PCN index (blue line) and 1-min variation, $|\mathrm{d} \boldsymbol{B} / \mathrm{d} t|$, in the horizontal geomagnetic field at Abisko (red) and Lovö (magenta line) around the time, 20:07 UT, of electric power disruption.

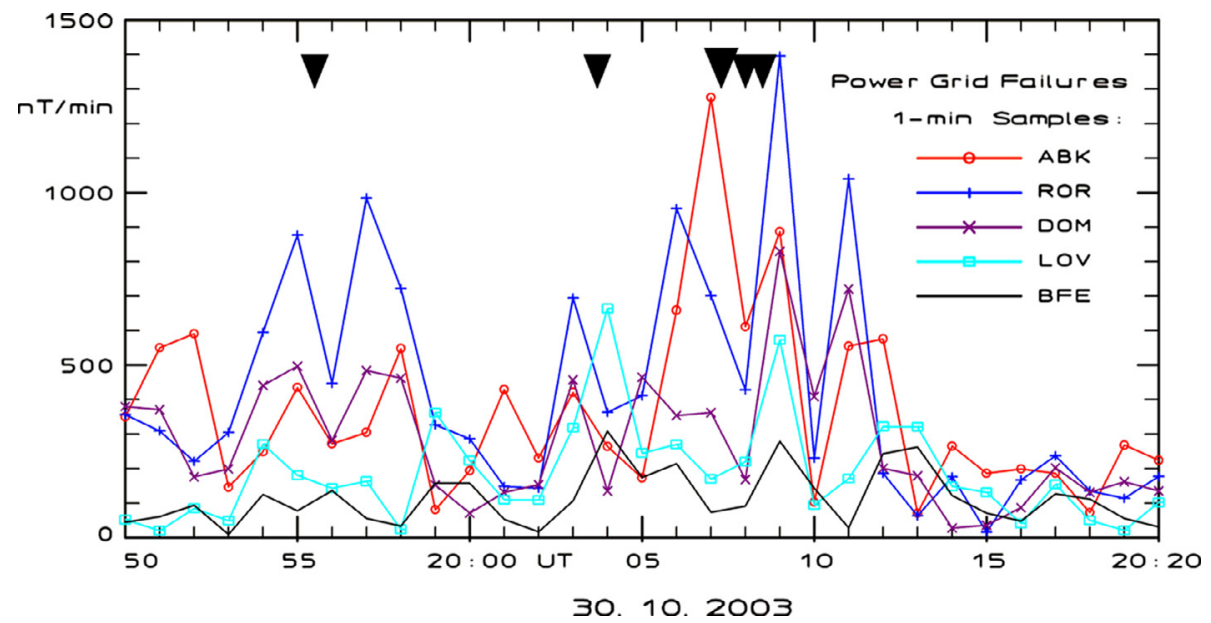

Figure 5. Detailed plot of 1-min variation, $|\mathrm{d} \boldsymbol{B} / \mathrm{d} t|$, in the horizontal magnetic field measured at various observatories in Scandinavia. Times of power grid problems are marked by the arrowheads.

still positioned in the afternoon sector (away from midnight) and the variability in the horizontal magnetic components at Lovö and Abisko is low or moderate. During the later PCN enhancement starting at 19:00 UT the Scandinavian Peninsula has reached the midnight sector of the auroral oval and the magnetic variability, $|\mathrm{d} \boldsymbol{B} / \mathrm{d} t|$, has increased considerably during $\sim$ 19:30-20:40 UT peaking at or close to 20:07 UT, the onset of Malmö power outage.

The more detailed distribution of magnetic variability vs. latitude in Scandinavia is evident from Figure 5. The observatories are located at invariant latitudes ranging between Abisko at $65.5^{\circ}$ and Brorfelde at $52.9^{\circ}$ invl. The other observatories are Rörvik at $62.6^{\circ}$, Dombås at $59.7^{\circ}$, and Lovö at $56.4^{\circ}$ invl. In Figure 5 the reported power grid disturbances (Pulkkinen et al. 2005) are marked by arrowheads with the largest one positioned at 20:07 for the onset of the power line outage in Malmö.

During the interval from 19:50 to 20:12 UT there are several strong magnetic variations. At 19:55 and 20:03 there were variations close to $600 \mathrm{nT} / \mathrm{min}$, that is, 1-min average variations of around $10 \mathrm{nT} / \mathrm{s}$, over most of Sweden giving power grid failures without serious consequences. At the time of the failure at 20:07 UT leading to the power outage in Malmö, the variability is largest at Abisko with a value of $1380 \mathrm{nT} / \mathrm{min}$, i.e. $23 \mathrm{nT} / \mathrm{s}$ (average), while in the preceding minute (20:06) the $\mathrm{d} B / \mathrm{d} t$ peaked at Rörvik at $960 \mathrm{nT} / \mathrm{min}$, i.e., $16 \mathrm{nT} / \mathrm{s}$ (average).

Either of these two enhancements may have triggered the high-voltage power line protective circuits to cause disruptions in Northern Sweden. In the southern part of Sweden the variability was fairly small at this time. How the disturbances of the high-voltage power line circuits in Northern Sweden were propagated to cause electric power outage in Malmö in Southern Sweden close to Brorfelde (BFE in Figs. 3 and 5) is a question for the power system management.

\section{Discussions}

Like noted above, the most significant parameter in control of GIC events is the horizontal magnetic field rate-of-change $(\mathrm{d} \boldsymbol{B} / \mathrm{d} t)$ value. The diagram in Figure 6 displays histograms of $\mathrm{d} B / \mathrm{d} t(|\mathrm{~d} \boldsymbol{B} / \mathrm{d} t|)$ values for Lovö through the stormy months reported here. The statistics is based on 1-min samples since data in this format is the only available source through all storms considered. When the $\mathrm{d} B / \mathrm{d} t$ values are given in the more handy nT/s unit, then these are averages through the 1-min 
P. Stauning: Power grid disturbances and polar cap index during geomagnetic storms
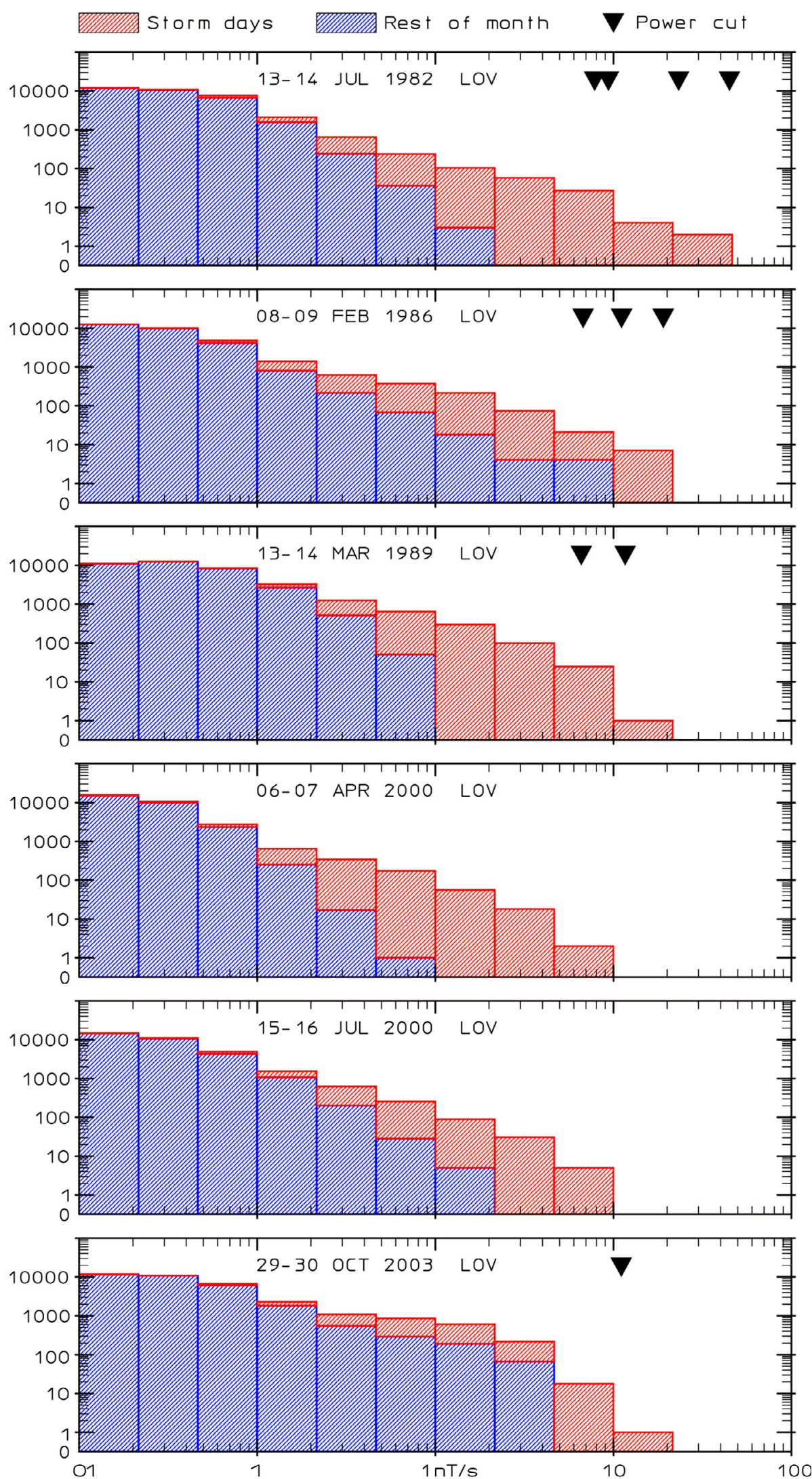

Figure 6. Monthly histograms of Lovö horizontal magnetic field time derivative $(\mathrm{d} B / \mathrm{d} t)$.

sample intervals. The heights of the columns indicate along the vertical axis the monthly number of occurrences of $\mathrm{d} B / \mathrm{d} t$ (1-min samples) within specific limits defined along the horizontal axis in 1-min average nT/s values. The solid (red) hatching displays the number of occurrences on the two most intense storm days. The light (blue) hatching denotes occurrences on the other days of the month. The triangles indicate $\max \mathrm{d} B / \mathrm{d} t$ values at Lovö at the time of power line disruptions. 
Table 1. List of max deviation and max time derivative for Lovo during magnetic storms.

\begin{tabular}{lcrc}
\hline \hline Storm period & $\operatorname{Max~d} B$ & $\operatorname{Max~d} B / \mathrm{d} t$ & Power cuts \\
\hline 13-14 July 1982 & $5381 \mathrm{nT}$ & $44.8 \mathrm{nT} / \mathrm{s}$ & 14 Reports \\
8-9 February 1986 & $2117 \mathrm{nT}$ & $21.2 \mathrm{nT} / \mathrm{s}$ & 5 Reports \\
13-14 March 1989 & $3007 \mathrm{nT}$ & $11.7 \mathrm{nT} / \mathrm{s}$ & 9 Reports \\
6-7 April 2000 & $1315 \mathrm{nT}$ & $7.2 \mathrm{nT} / \mathrm{s}$ & No reports \\
15-16 July 2000 & $1252 \mathrm{nT}$ & $6.6 \mathrm{nT} / \mathrm{s}$ & No reports \\
29-30 October 2003 & $2423 \mathrm{nT}$ & $11.1 \mathrm{nT} / \mathrm{s}$ & Malmö outage \\
\hline
\end{tabular}

They are clearly positioned at the upper end of the distributions for the 1982, 1986, 1989, and 2003 cases. During the two very strong magnetic storms in 2000 , which have been added to the display, the $\mathrm{d} B / \mathrm{d} t$ values did not reach the level required for power line tripping and there are no reports on high-voltage power grid failures during these two storms.

The maximum deviation, $\mathrm{d} B$, from the quiet level and the maximum rate-of-change, $\mathrm{d} B / \mathrm{d} t$, values, based on 1-min samples of the horizontal components, reached at Lovö during the storms, are given in Table 1 . The $\mathrm{d} B / \mathrm{d} t$ numbers in $\mathrm{nT} / \mathrm{s}$ units are average values through the sample interval.

The adverse GIC events that affect power grids are clearly associated with fast and deep geomagnetic variations. The reported events (all events reported here) are mainly related to tripping of protection circuits and not to equipment (HV transformer) damage. It is worth noting that the GIC currents by themselves could not possibly overheat transformers. They could shift the operating base magnetism of the core such that the core may enter saturation in one or the other half-wave phase of the operating $\mathrm{AC}$ current if the transformer is operated close to its limits. Tripping of protective circuits during GIC events is probably unavoidable. However, overheating of transformers, which is the most alarming GIC effect, should be avoided.

Predictions of the strength and time variations of magnetic substorms have not yet reached a mature level for practical applications although post-event analyses have reached a high level of accuracy (e.g., Wik et al. 2008, 2009). General warnings of magnetic storm conditions following solar outbursts (CMEs) are provided by satellite data. Local magnetic data can clearly define the onset and the further development of substorms but they may not provide advance warnings. The actual substorm onset could be triggered by plasma instabilities in the tail region or by sudden changes in the solar wind plasma parameters or magnetic fields - even by a northward turning. The possibly best indication of imminent substorm activity is provided by using on-line PC indices to enable monitoring of polar cap convection building stresses in the magnetotail that could be released in strong substorms. In addition, the duration of the enhanced PC level is important for judging the possible equatorward displacement of substorm activity. As illustrated in Figure 7 the HV line disruption events reported here were preceded by PCN index values at or exceeding 10 units through one or more hours before the stroke. For the stronger cases in 1982, 1989, and 2003, the PCN index values exceeded 20 units during the hour preceding the disturbance.

To set this number in perspective to see how frequent such occurrences are, Figure 8 displays the yearly number of hours with PCN values consistently above limits of 10,15 , and 20 units. At PCN above 10 units there is a considerable risk of strong substorms. When the hourly PCN value exceeds the level 20 units there is an imminent risk of the occurrence of very strong substorms displaced to unusually low latitudes, which may seriously endanger vital electric power grids.

In Figure 8 the 11-year solar cycle variations in the occurrence frequencies are seen. During solar minimum the strong events are almost absent. It should be noted that the very strong events with hourly PCN values above 15 or 20 units are quite sparse - totally only a few hours a year - even during solar maximum years. This feature is important for the possible use of an on-line PCN index for warning of adverse space weather since too frequent alarming may reduce the alertness.

\section{Conclusions}

In all events reported here the power line systems disturbed by GIC effects were located in the middle or southern part of Sweden at geomagnetic invariant latitudes around $55-60^{\circ}$, i.e. in the sub-auroral zone. In this region the time variations of geomagnetic disturbances are often faster than in auroral and polar regions. During the great geomagnetic storms the amplitudes of disturbances here are similar to or may exceed those normally found at higher latitudes. Hence the time derivatives may greatly exceed values which could be considered typical of disturbances observed at basic auroral and polar latitudes.

- It was further noted that disrupts of power transmission circuits occurred when the rate of change in the geomagnetic field, $\mathrm{d} B / \mathrm{d} t$, in Mid-Sweden exceeded around $10 \mathrm{nT} / \mathrm{s}$ based on 1-min samples. This value is not an absolute threshold since, among other, the orientation and extent of the primary ionospheric currents relative to the power grid are very important. One example was the 30 October 2003 event when the peak ionospheric current surge which was considered responsible for the power outage in Malmö was oriented along the length axis of Sweden to produce maximum effect on North-South going high-voltage power lines.

- All here reported power line disrupts were associated with major geomagnetic storms resulting from large solar outbursts (CME). They occurred during solar maximum $(1982,1989)$ as well as intermediate (2003) and solar minimum (1986) conditions. With modern CME observations these storms, most likely, could be predicted 1 or 2 days in advance to the benefit of power grid managements. GIC events are particularly likely to occur at the arrival of CME clouds and then during intense substorms in the following days. The arrival of a CME cloud to the Earth can be predicted 30-60 min in advance from near-Earth monitoring satellites, e.g. the ACE satellite at the L1 point. However, the exact time of occurrence of the events associated with onset of substorms cannot be predicted accurately, yet.

- An on-line PC index could be used to provide early warning of the build-up of magnetospheric stresses that in turn could be released in violent substorms endangering the electric power grids. The duration of enhanced PC index levels is important for judging the equatorward displacement of anticipated substorm activity. All cases considered here were preceded by strongly enhanced PC index values at or above 10 units at least $30-60 \mathrm{~min}$ in advance. For the stronger cases the average PC index exceeded 20 units during the hour preceding the disturbance event. This situation happens at most a few hours a year even during solar maximum conditions. 
P. Stauning: Power grid disturbances and polar cap index during geomagnetic storms

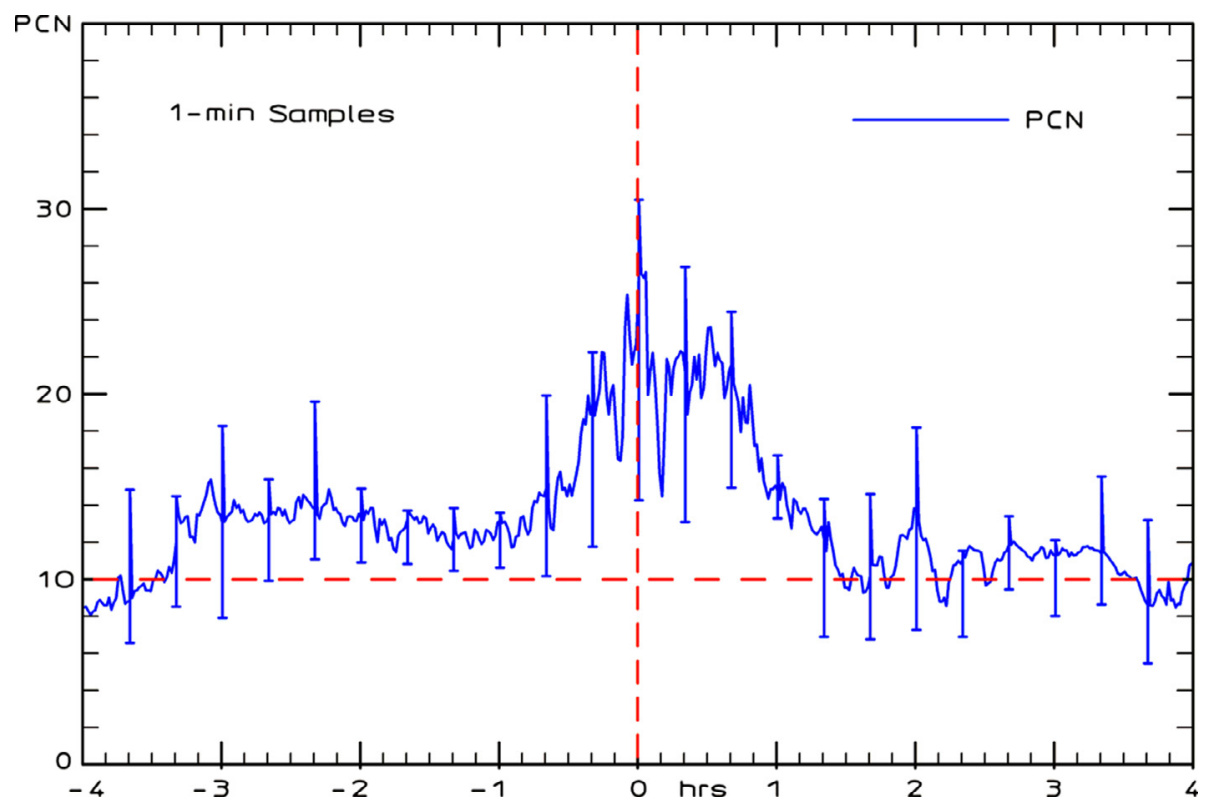

Figure 7. Average PCN index values during $4 \mathrm{~h}$ preceding and $4 \mathrm{~h}$ following the power grid disruptions reported in Figure 2 . Standard deviation ranges are marked.

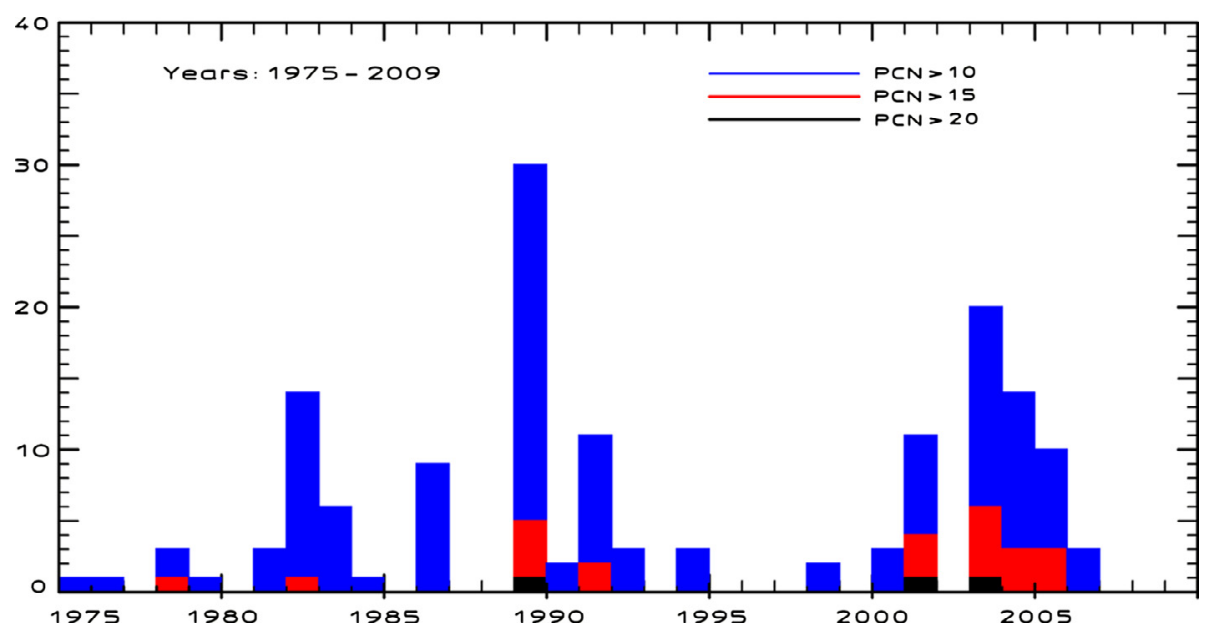

Figure 8. Yearly number of hours of PCN values consistently above levels of 10, 15, and 20 units.

- The PC index should not be considered a replacement of other early warning systems based, for instance, on monitoring solar wind conditions from interplanetary spacecrafts like the ACE satellite. But rather as a supplement with two important features. The real-time monitoring of the polar cap convection provides a realistic measure of the terrestrial effects of solar wind enhancements and may help to avoid false alerts. In addition the real-time PC index could be considered a back-up system to be used in case the interplanetary satellite(s) are disabled by technical problems or possibly harmed by the intense highenergy solar proton radiation, which often accompany the really strong solar eruptions.

- Trippings of line protection circuits during GIC events are probably unavoidable. The management of power grids should minimize consequences and provide quick restoration. Overheating of transformers is avoidable. At times of possible strong substorm activity and in the GIC risk zone transformers with ground connections should be operated with caution. The rugged design of transformers and protective circuits is imperative.

Acknowledgements. The information on high-voltage power grid disturbances supplied from Vattenfallet Electric Power Company is most gratefully acknowledged. The collection and availability of geomagnetic data recorded at the DMI observatory in Qaanaaq (Thule) forming the basis for the PCN index and the geomagnetic data from the observatories in Abisko, Lovö, Brorfelde, and other magnetic observatories, supplied through the World Data Centre in Kyoto, Japan, are gratefully acknowledged.

\section{References}

Boteler, D.H., R.J. Pirjola, and H. Nevanlinna, The effects of geomagnetic disturbances on electrical systems at the Earth's surface, Adv. Space Res., 22 (1), 17-27, 1998. 
Janzhura, A., O. Troshichev, and P. Stauning, Unified PC indices: Relation to isolated magnetic substorms, J. Geophys. Res., 112, A09207, DOI: 10.1029/2006JA012132, 2007.

Kan, J.R., and L.C. Lee, Energy coupling function and solar windmagnetosphere dynamo, Geophys. Res. Lett., 6 (7), 577-580, 1979.

Kappenman, J., Geomagnetic Storms and their Impacts on the U.S. Power Grid, Metatech Report, Meta-R-319, 197 pp, 2010 (http://www.ornl.gov./sci/ees/etsd/pes/pubs/ferc_Meta-R-319.pdf).

Pirjola, R., Modelling the electric and magnetic fields at the Earth's surface due to an auroral electrojet, J. Atmos. Sol. Terr. Phys., 60, 1139-1148, 1998.

Pirjola, R. and A. Viljanen, Complex image method for calculating electric and magnetic fields produced by an auroral electrojet of finite length, Ann. Geophys., 16, 1434-1444, 1998

Pulkkinen, A., S. Lindahl, A. Viljanen, and R. Pirjola, Geomagnetic storm of 29-31 October 2003: Geomagnetically induced currents and their relation to problems in the Swedish high-voltage power transmission system, Space Weather, 3, S08C03, DOI: 10.1029/2004SW000123, 2005.

Stauning, P., Modelling electrojet currents causing power line disturbances during large geomagnetic storms, in: R. Gendrin et al. (eds.) ESTEC Proceedings from Space Weather Workshop 1719 December 2001: Looking towards a European Space Weather Program, 223-226, 2001.

Stauning, P., Comment on "The PC index: review of methods" by McCreadie and Menvielle (2010), Ann. Geophys., 29, 1137-1146, 2011.

Stauning, P., The polar cap PC indices. Relations to solar wind and global disturbances, in: M. Lazar (ed.), Exploring the Solar Wind, Intech Publ. Co., ISBN: 978-953-51-339-4, DOI: 10.5772/37359, 2012.

Stauning, P., and J. Watermann, High-voltage power-line disturbances and electrojet modelling during large geomagnetic storms, in: H. Sawaya-Lacoste (ed.), Proceedings from SUNSPA Conference 2001, ESA SP477, 521-524, 2002.

Stauning, P., O. Troshichev, and A. Janzhura, The polar cap (PC) index. Relations to solar wind parameters and global magnetic activity level, J. Atmos. Sol. Terr. Phys., 70, 2246-2261, DOI: 10.1016/j.jastp.2008.09.028, 2008.

Thomson, A.W.P., E.B. Dawson, and S.J. Reay, Quantifying extreme behaviour in geomagnetic activity, Space Weather, 9, S10001, DOI: 10.1029/2011SW000696, 2011.

Troshichev, O.A., and V.G. Andrezen, The relationship between interplanetary quantities and magnetic activity in the southern polar cap, Planet. Space Sci., 33, 415-419, 1985.

Troshichev, O.A., V.G. Andrezen, S. Vennerstrom, and E. FriisChristensen, Magnetic activity in the polar cap - A new index, Planet. Space Sci., 36, 1095-1102, 1988.

Troshichev, O.A., N.P. Dmitrieva, and B.M. Kuznetsov, Polar cap magnetic activity as a signature of substorm development, Planet. Space Sci., 27, 217-221, 1979.

Troshichev, O., A. Janzhura, and P. Stauning, Unified PCN and PCS indices: method of calculation, physical sense, and dependence on the IMF azimuthal and northward components, J. Geophys. Res., 111, A05208, 10 pp, DOI: 10.1029/2005JA011402, 2006.

Vennerstrøm, S., The geomagnetic activity index PC, PhD Thesis, Scientific Report 91-3, Danish Meteorological Institute, 105 pp, 1991.

Viljanen, A., and R. Pirjola, Statistics on geo-magnetically-induced currents in the finnish $400 \mathrm{kV}$ power system based on recordings of geomagnetic variations, J. Geomag. Geoelectr., 41, 411-420, 1989.

Viljanen, A., O. Amm, and R. Pirjola, Modelling geomagnetically induced currents during different ionospheric situations, $J$. Geophys. Res., 104, 28059-28071, 1999.

Viljanen, A., H. Nevanlinna, K. Pajunpää, and A. Pulkkinen, Time derivative of the horizontal geomagnetic field as an activity indicator, Ann. Geophys., 19, 1107-1118, 2001.

Wik, M., A. Viljanen, R. Pirjola, A. Pulkkinen, P. Wintoft, and H. Lundstedt, Calculation of Geomagnetically Induced Currents in the $400 \mathrm{kV}$ Power System in Southern Sweden, Space Weather, 6, S07005, DOI: 10.1029/2007SW000343, 2008.

Wik, M., R. Pirjola, H. Lundstedt, A. Viljanen, P. Wintoft, and A.A. Pulkkinen, Space weather effects in July 1982 and October 2003 and the effects of geomagnetically induced currents on Swedish technical systems, Ann. Geophys., 27, 1775-1787, 2009.

Cite this article as: Stauning P: Power grid disturbances and polar cap index during geomagnetic storms. J. Space Weather Space Clim., 2013, 3, A22. 